\title{
CRITERIONS FOR DETECTABILITY AND STRONG DETECTABILITY OF FAULTS IN LINEAR SYSTEMS
}

\author{
Mattias Nyberg
}

\author{
Department of Electrical Engineering, Linköping University \\ SE-58183 Linköping, Sweden \\ Email: matny@isy.liu.se
}

\begin{abstract}
A fault is (strongly) detectable if it is possible to construct a residual generator that is sensitive to the (constant) fault while decoupling all disturbances. Two new fault detectability criterions and three new strong fault detectability criterions are presented. For comparison, existing fault detectability criterions are reviewed. To prove the criterions, a framework of polynomial bases is utilized. With the set of new criterions, together with the previously known, there exists now a criterion for models given both on transfer function form and state-space form, and for both fault detectability and strong fault detectability investigations. Recommendations are given on what criterion to use in different situations.
\end{abstract}

Keywords: Diagnosis, Fault Detection, Residual Generation, Fault Detectability, Detectability Criterions

\section{INTRODUCTION}

A fundamental part of a model based fault diagnosis system is the residual generator. The residual generator filters the known signals and generates a signal, the residual, that should be small (ideally 0 ) in the fault-free case and large when a fault is acting on the system. In a general system, not only the control signal $u$ influence the system, but also disturbances $d$ and the faults $f$. Both faults and disturbances will in this paper be assumed to be modeled as signals.

The topic of this paper is fault detectability, or more exactly, if it is possible to construct a residual generator that is sensitive to a certain fault $f$ while decoupling all disturbances $d$. Only linear systems will be considered. Detectability of faults that are modeled as constant signals are explicitly investigated. Such detectability is usually called strong fault detectability,

Criterions for both fault detectability and strong fault detectability are derived. In Section 4, two new fault detectability criterions are derived. For comparison, also existing criterions are reviewed. In Section 5, three new strong fault detectability criterions are derived. Finally, Section 6 contains a comparison of the different criterions.

\section{PRELIMINARIES}

For simplicity reasons, we will only discuss the continuous case. However, corresponding results for the discrete case can be derived in a similar manner. Throughout this paper, we will assume that the fault signal $f(t)$ is a scalar signal. This makes most sense since we are interesting in checking detectability with respect to one particular fault.
We assume that the model of the system is given either in the transfer function form

$$
y=G(s) u+H(s) d+L(s) f
$$

or in the state-space form

$$
\begin{aligned}
& \dot{x}(t)=A x(t)+B_{u} u(t)+B_{d} d(t)+B_{f} f(t) \\
& y(t)=C x(t)+D_{u} u(t)+D_{d} d(t)+D_{f} f(t)
\end{aligned}
$$

The dimension of $y(t)$ is $m, u(t)$ is $k_{u}$, and $d(t)$ is $k_{d}$.

This paper relies on established theory on polynomial matrices, polynomial/rational vector spaces, and polynomial bases for these spaces (Kailath, 1980; Forney, 1975; Chen, 1984). We will use the notation $\operatorname{Im} A(s)$ to denote the column image (also called the column range) of a matrix $A(s)$, and the notation $\mathcal{N}_{L}\{A(s)\}$ to denote the left null-space.

\subsection{Residual Generators}

A general linear residual generator can be written

$$
r=Q(s)\left(\begin{array}{l}
y \\
u
\end{array}\right)
$$

i.e. $Q(s)$ is a multi-dimensional transfer matrix with known signals $y$ and $u$ as inputs and a residual as output.

Inserting (1) into (3) gives

$$
r=Q(s)\left[\begin{array}{cc}
G(s) & H(s) \\
I & 0
\end{array}\right]\left[\begin{array}{l}
u \\
d
\end{array}\right]+Q(s)\left[\begin{array}{c}
L(s) \\
0
\end{array}\right] f
$$

Define $M(s)$ as

$$
M(s)=\left[\begin{array}{cc}
G(s) & H(s) \\
I & 0
\end{array}\right]
$$

To make $r(t)=0$ when $f(t)=0$, it is required that disturbances and the control signal are decoupled, i.e. 
for $Q(s)$ to be a residual generator, it must hold that $Q(s) M(s)=0$. To be able to detect faults, it is also required that the residual is sensitive to faults. This requirement can be expressed as

$$
Q(s)\left[\begin{array}{c}
L(s) \\
0
\end{array}\right] \neq 0
$$

Thus, design of a residual generator consists of finding a matrix $Q(s)$ that fulfills two requirements: belongs to the left null-space of $M(s)$ and fulfills (5).

Assume that the rows of a matrix $N_{M}(s)$ form an irreducible (i.e. full rank for all $s$ ) basis for the left null-space of $M(s)$. Then it is shown in (Nyberg and Frisk, 1999) that all stable residual generators $r$ can be parameterized as $r=c^{-1}(s) \phi(s) N_{M}(s)\left[y^{T} u^{T}\right]^{T}$, where $c(s)$ is a scalar polynomial with its roots in the left half-plane and $\phi(s)$ is a polynomial vector. How to find such a basis $N_{M}(s)$ is not the topic of this paper but procedures for this can be found in (Nyberg and Frisk, 1999).

\section{FAULT DETECTABILITY AND STRONG FAULT DETECTABILITY}

Fault detectability and strong fault detectability, were in (Chen and Patton, 1994) defined as properties of a residual generator. Here, fault detectability and strong fault detectability are instead defined as properties of the system:

Definition 1. (Fault Detectability ). A fault $f$ is detectable in a system if there exists a residual generator such that the transfer function from the fault to the residual is nonzero, i.e. $G_{r f}(s) \neq 0$, and the transfer functions from the known input $u$ and the disturbance $d$ to the residual are zero, i.e. $G_{r u}(s)=0$ and $G_{r d}(s)=0$.

Definition 2. (Strong Fault Detectability). A fault $f$ is strongly detectable in a system if there exists an asymptotically stable residual generator such that the transfer function from the fault to the residual $G_{r f}(s)$ has a nonzero DC-gain, i.e. $G_{r f}(0) \neq 0$, and the transfer functions from the known input $u$ and the disturbance $d$ to the residual are zero, i.e. $G_{r u}(s)=0$ and $G_{r d}(s)=0$.

Strong fault detectability is important since many faults are constant when present. If such a fault is not strongly detectable, then its affect on the residual is only a short pulse and therefore more difficult to detect. Faults that are detectable but not strongly detectable will be called weakly detectable faults.

In some particular cases, it can be quite simple to show that a fault is for example only weakly detectable. However, in the general case, this is much more difficult. Therefore it is useful to have general criterions for both fault detectability and strong fault detectability. Such criterions are developed in the next two sections.

\section{DETECTABILITY CRITERIONS}

In this section, four general detectability criterions are presented. The first two criterions assume that the system is given on the transfer function form (1) and the next two criterions assume that the system is given on the state space form (2). Due to the use of polynomial bases, the analysis becomes quite simple. The benefit of polynomial bases will be even more clear in the next section, where strong fault detectability is investigated.

Note that even though three of the criterions in this section have been presented and partly or fully proven before (see references below), it is useful to review them here. The reason is that by presenting them here, all in the same framework and with the same notation, it becomes easy to compare them and get an good overview. In addition, it is also interesting to later compare each criterion for fault detectability with the corresponding criterion for strong fault detectability.

\subsection{The Intuitive Approach}

From the discussion on residual generation in Section 2.1, it follows, quite intuitively, that the following theorem is a criterion for fault detectability:

Theorem 1. A fault $f$ is detectable in a system if and only if

$$
\operatorname{Im}\left[\begin{array}{c}
L(s) \\
0
\end{array}\right] \nsubseteq \operatorname{Im}\left[\begin{array}{cc}
G(s) & H(s) \\
I & 0
\end{array}\right]
$$

For a proof, see (Nyberg, 1999).

The easiest way to check condition (6) is probably by studying the rank as follows: a fault is detectable if and only if

$$
\operatorname{Rank}\left[\begin{array}{ccc}
G(s) & H(s) & L(s) \\
I & 0 & 0
\end{array}\right]>\operatorname{Rank}\left[\begin{array}{cc}
G(s) & H(s) \\
I & 0
\end{array}\right]
$$

It is obvious that this rank-condition is equivalent to $(6)$.

A second alternative to check condition (6) is to calculate a basis for $\mathcal{N}_{L}\{M(s)\}$, i.e. the left nullspace of $M(s)$. Let the rows of a matrix $N_{M}(s)$ form a basis for $\mathcal{N}_{L}\{M(s)\}$. Then we have that a fault is detectable if and only if

$$
N_{M}(s)\left[\begin{array}{c}
L(s) \\
0
\end{array}\right] \neq 0
$$

However, to calculate a basis for the null-space requires more involved algorithms than a rank test.

Remark: Both the calculation of the rank of a rational matrix and a basis for the left (or right) null-space, can be done by first performing an MFD. Consider for example an MFD $G(s)=N(s) D^{-1}(s)$. Since the column ranges of $G(s)$ and $N(s)$ are equal, the rank and a basis for the left null-space of $G(s)$ can be calculated by instead considering the polynomial matrix $N(s)$. The rank, and a basis for the nullspace, of $N(s)$ can then easily be calculated by using procedures implemented in The Polynomial Toolbox 2.0 for Matlab 5 (1998).

\subsection{The "Frequency Domain" Approach}

Here we will review a somewhat simpler, but closely related, alternative to Theorem 1 . This criterion is well known and was given in e.g. (Ding and Frank, 1991). 
Theorem 2. A fault $f$ is detectable in a system if and only if

$$
\operatorname{Im} L(s) \nsubseteq \operatorname{Im} H(s)
$$

It is easy to prove that this theorem is equivalent to Theorem 1. However, this criterion is much simpler, since it does not include $G(s)$. Also here, the check can be performed by doing a rank test or to calculate a basis for the null space.

\subsection{Using the System Matrix}

When the system model is available in statespace form, the system matrix in state-space form (Rosenbrock, 1970) can be used to check fault detectability. Similar conditions for fault detectability were noted in for example (Magni and Mouyon, 1994).

Theorem 3. A fault $f$ is detectable in a system if and only if

$$
\operatorname{Im}\left[\begin{array}{l}
D_{f} \\
B_{f}
\end{array}\right] \nsubseteq \operatorname{Im}\left[\begin{array}{cc}
C & D_{d} \\
A-s I & B_{d}
\end{array}\right]
$$

A proof can be found in (Nyberg, 1999).

\subsection{Using the Chow-Willsky Scheme}

The criterion given here is new and based on the Chow-Willsky scheme (Chow and Willsky, 1984; Frank, 1990). Again we assume that the system is given on the state-space form (2).

Before the criterion can be presented, some notation from the Chow-Willsky scheme is reviewed. Designing residual generators with the Chow-Willsky scheme comes down to finding a vector $w$ in the left null-space of a constant matrix $\left[\mathcal{R}_{\rho} \mathcal{H}_{\rho}\right]$, where $\mathcal{R}_{\rho}=\left[\begin{array}{llll}C^{T} & A^{T} C^{T} & \ldots & A^{\rho T} C^{T}\end{array}\right]^{T}$ and $\mathcal{H}_{\rho}$ is a lower triangular Toeplitz matrix describing the propagation of the disturbances through the system. The constant $\rho$ is determined by the user. Further, $\mathcal{Q}_{\rho}$ is a lower triangular Toeplitz matrix describing the propagation of the inputs $u$ through the system. Similarly, $\mathcal{P}_{\rho}$ is a lower triangular Toeplitz matrix describing the propagation of the fault $f$ through the system.

Theorem 4. A fault $f$ is detectable in a system if and only if

$$
\operatorname{Im} \mathcal{P}_{n} \nsubseteq \operatorname{Im}\left[\mathcal{R}_{n} \mathcal{H}_{n}\right]
$$

The proof can be found in (Nyberg, 1999) but it has similarities with the proof of Theorem 7 presented later. Theorem 4 might seem trivial at first, but when constructing a proof, two difficulties arise. The first is that the pair $\left\{A,\left[B_{u} B_{d}\right]\right\}$ may not be controllable, which has the consequense that not all residual generators can be constructed with the Chow-Willsky scheme, see (Nyberg and Nielsen, 1997). The second is to show that the number $\rho$ can be chosen as $\rho=n$, where $n$ is the order of the realization (2).

Remark: In design of residual generators, using a model on state-space form, it is important to ensure that the pair $\left\{A,\left[B_{u} B_{d}\right]\right\}$ is controllable, see (Nyberg and Nielsen, 1997; Nyberg and Frisk, 1999).
As is seen in both Theorem 3 and 4, this is not the case when checking detectability.

\section{STRONG DETECTABILITY CRITERIONS}

It is well known that faults often become weakly detectable when the system contains an integration. However, faults can be weakly detectable also if the system do not contain an integration. This can for example be seen in Example 1 below. Further, it is easy to show that poles in the origin will not necessarily lead to that a fault becomes weakly detectable. Thus, the problem of checking strong fault detectability is more involved than only checking the existence of poles in the origin. Below we will investigate if there are any similar criterions, corresponding to the four criterions given in Section 4, that are general criterions for strong fault detectability.

When checking fault detectability with Theorem 1, it was noted that there are at least three options: condition (6) could be used directly, a rank test (7) could be performed, or a basis for $\mathcal{N}_{L}\{M(s)\}$ could be calculated as in (8). All these three options are also possible for the other detectability criterions, i.e. Theorem 2, 3, and 4. When checking strong detectability using rational or polynomial matrices, there is only one option; a basis for $\mathcal{N}_{L}\{M(s)\}$ must be calculated. The underlying reason why a condition of the type (6) (or (7)) can not be used, i.e. by replacing $s$ with 0 , is the following. The important thing for checking detectability is $\operatorname{Im} N_{M}(s)$, and for strong detectability $\operatorname{Im} N_{M}(0)$. While it holds that

$$
\operatorname{Im} N_{M}(s)=\mathcal{N}_{L}\{M(s)\}
$$

we can not find $\operatorname{Im} N_{M}(0)$ via $\mathcal{N}_{L}\{M(0)\}$ since in general,

$$
\operatorname{Im} N_{M}(0) \neq \mathcal{N}_{L}\{M(0)\}
$$

This will be further illustrated in Example 1, included in the next section below.

In the presentation of the theorems for strong detectability, the notation $\left.(\cdot)\right|_{s \rightarrow 0}$ will be used, with the meaning $\left.A(s)\right|_{s \rightarrow 0}=\lim _{s \rightarrow 0} A(s)$. Note that this is the same as to first carry out all cancellations, and then substitute $s$ with 0 .

\subsection{The Intuitive Approach}

The criterion corresponding to Theorem 1 becomes:

Theorem 5. A fault $f$ is strongly detectable in a system if and only if

$$
\left.\left(N_{M}(s)\left[\begin{array}{c}
L(s) \\
0
\end{array}\right]\right)\right|_{s \rightarrow 0} \neq 0
$$

where the rows of $N_{M}(s)$ are an irreducible polynomial basis for $\mathcal{N}_{L}\{M(s)\}$.

The proof can be found in (Nyberg, 1999).

Example 1. Consider a system described by the following transfer functions:

$G(s)=\left[\begin{array}{c}\frac{1}{s+1} \\ \frac{1}{s+1}\end{array}\right] \quad H(s)=\left[\begin{array}{c}\frac{s}{s+2} \\ \frac{s+2}{s+2}\end{array}\right] \quad L(s)=\left[\begin{array}{l}\frac{s+1}{s+3} \\ \frac{1}{s+3}\end{array}\right]$ 
Then a right MFD of the matrix $M(s)$ is

$$
M(s)=\left[\begin{array}{cc}
G(s) & H(s) \\
I & 0
\end{array}\right]=\left[\begin{array}{cc}
1 & s \\
1 & s \\
s+1 & 0
\end{array}\right]\left[\begin{array}{cc}
s+1 & 0 \\
0 & s+2
\end{array}\right]^{-1}
$$

A minimal polynomial basis for the left null-space of $M(s)$ is $[1-10]$. By using Theorem 5 , the check for strong fault detectability becomes

$$
\left.\left[\begin{array}{lll}
1 & -1 & 0
\end{array}\right]\left[\begin{array}{c}
L(s) \\
0
\end{array}\right]\right|_{s \rightarrow 0}=\left.\frac{s}{s+3}\right|_{s \rightarrow 0}=0
$$

and the fault is therefore not strongly detectable. Note that the fault is not strongly detectable even though the system has no poles in the origin.

Now we will show that it is not sufficient to consider the left null-space of $M(0)$. A minimal polynomial basis for $\mathcal{N}_{L}\{M(0)\}$ is

$$
\left[\begin{array}{ccc}
1 & -1 & 0 \\
0 & 1 & -1
\end{array}\right]
$$

The check for strong fault detectability would be

$$
\left.\left[\begin{array}{ccc}
1 & -1 & 0 \\
0 & 1 & -1
\end{array}\right]\left[\begin{array}{c}
L(s) \\
0
\end{array}\right]\right|_{s \rightarrow 0}=\left.\left[\begin{array}{c}
\frac{s}{s+3} \\
\frac{1}{s+3}
\end{array}\right]\right|_{s \rightarrow 0}=\left[\begin{array}{l}
0 \\
\frac{1}{3}
\end{array}\right] \neq 0
$$

which wrongly indicates that the fault is strongly detectable. This means that a condition for strong fault detectability can not be based on a basis for $\mathcal{N}_{L}\{M(0)\}$.

\subsection{The "Frequency Domain" Approach}

We have concluded that a basis for the null-space must be calculated to check strong fault detectability. However, even if we do so, the "frequency domain" approach, from Section 4.2, will not work. This is shown in the following example:

Example 2. Consider a system described by the following transfer functions:

$$
G(s)=\left[\frac{1}{s} \frac{1}{s}\right]^{T} \quad H(s)=\left[\frac{1}{s} 1\right]^{T} \quad L(s)=\left[\begin{array}{ll}
0 & 1
\end{array}\right]^{T}
$$

By using Theorem 5, it can be concluded that the fault is not strongly detectable. Now the question is if we can use a condition for strong fault detectability based on (9) if we actually calculate an irreducible basis $N_{H}(s)$ for the left null-space to $H(s)$. In other words, is $\left.\left(N_{H}(s) L(s)\right)\right|_{s \rightarrow 0} \neq 0$ a condition for strong fault detectability? To check this, we calculate a basis $N_{H}(s)$, which becomes $[s-1]$. Then we have that

$$
\left.\left(N_{H}(s) L(s)\right)\right|_{s \rightarrow 0}=\left.[s-1]\left[\begin{array}{c}
0 \\
-1
\end{array}\right]\right|_{s \rightarrow 0}=1 \neq 0
$$

which wrongly indicates that the fault is strongly detectable. This means that $\left.\left(N_{H}(s) L(s)\right)\right|_{s \rightarrow 0} \neq 0$ is not a condition for strong detectability.

\subsection{Using the System Matrix}

The criterion for strong fault detectability, corresponding to Theorem 3, becomes as follows:
Theorem 6. A fault $f$ is strongly detectable in a system if and only if

$$
N_{M_{s}}(0)\left[\begin{array}{l}
D_{f} \\
B_{f}
\end{array}\right] \neq 0
$$

where the rows of $N_{M_{s}}(s)$ are a basis for the left null-space of the matrix

$$
M_{s}(s)=\left[\begin{array}{cc}
C & D_{d} \\
-s I+A & B_{d}
\end{array}\right]
$$

To prove this theorem, we first need the following lemma.

Lemma 1. A fault $f$ is strongly detectable in a system if and only if

$$
\left.\left(N_{M}(s)\left[\begin{array}{c}
L(s) \\
0
\end{array}\right]\right)\right|_{s \rightarrow 0} \neq 0
$$

where the rows of $N_{M}(s)$ are a polynomial basis for $\mathcal{N}_{L}\{M(s)\}$ and $N_{M}(0)$ has full row-rank.

PROOF. The basis $N_{M}(s)$ can be written $N_{M}(s)=$ $R(s) N_{M}^{i r r}(s)$, where $R(s)$ is a greatest common divisor with full rank and $N_{M}^{i r r}(s)$ is an irreducible basis. Since $N_{M}(0)$ has full row-rank, $R(0)$ must have full rank. It can be shown that also $\left.R^{-1}(s)\right|_{s \rightarrow 0}$ must have full rank.

The condition (12) for strong fault detectability can be written as

$$
\begin{aligned}
& 0=\left.\left(N_{M}^{i r r}(s)\left[\begin{array}{c}
L(s) \\
0
\end{array}\right]\right)\right|_{s \rightarrow 0}= \\
& =\left.\left.\left.R^{-1}(s)\right|_{s \rightarrow 0} R(s)\right|_{s \rightarrow 0}\left(N_{M}^{i r r}(s)\left[\begin{array}{c}
L(s) \\
0
\end{array}\right]\right)\right|_{s \rightarrow 0}= \\
& =\left.\left.R^{-1}(s)\right|_{s \rightarrow 0}\left(R(s) N_{M}^{i r r}(s)\left[\begin{array}{c}
L(s) \\
0
\end{array}\right]\right)\right|_{s \rightarrow 0}= \\
& =\left.\left.R^{-1}(s)\right|_{s \rightarrow 0}\left(N_{M}(s)\left[\begin{array}{c}
L(s) \\
0
\end{array}\right]\right)\right|_{s \rightarrow 0}
\end{aligned}
$$

Since $\left.R^{-1}(s)\right|_{s \rightarrow 0}$ has full rank, this condition is equivalent to

$$
\left.\left(N_{M}(s)\left[\begin{array}{c}
L(s) \\
0
\end{array}\right]\right)\right|_{s \rightarrow 0}=0
$$

The negation of this condition is then equivalent to (13), which proves the lemma.

Now return to the proof of Theorem 6 :

PROOF. Let the row vectors of $V(s)$ be a polynomial basis for $\mathcal{N}_{L}\left\{M_{s}(s)\right\}$ and form $W(s)=V(s) P$, where $P$ is

$$
P=\left[\begin{array}{ll}
I & -D_{u} \\
0 & -B_{u}
\end{array}\right]
$$

According to Theorem 1 in (Nyberg and Frisk, 1999), the rows of $W(s)$ form a polynomial basis for $\mathcal{N}_{L}\{M(s)\}$. Without loss of generality, we can assume that the realization (2) is partitioned as follows: 


$$
\begin{aligned}
{\left[\begin{array}{c}
\dot{x} \\
\dot{z}
\end{array}\right]=} & {\left[\begin{array}{cc}
A_{x} & A_{12} \\
0 & A_{z}
\end{array}\right]\left[\begin{array}{l}
x \\
z
\end{array}\right]+\left[\begin{array}{c}
B_{u, x} \\
0
\end{array}\right] u+} \\
& {\left[\begin{array}{c}
B_{d, x} \\
0
\end{array}\right] d+\left[\begin{array}{l}
B_{f, x} \\
B_{f, z}
\end{array}\right] f } \\
y= & {\left[C_{x} C_{z}\right]\left[\begin{array}{l}
x \\
z
\end{array}\right]+D_{u} u+D_{d} d+D_{f} f }
\end{aligned}
$$

where the state $x$ is controllable from $\left[u^{T} d^{T}\right]^{T}$ and the state $z$ is controllable from the fault $f$. Assume also that the state $z$ is asymptotically stable, which is the same as saying that the whole system is stabilizable. Now note that

$$
\begin{aligned}
& {[W(s) 0]=V(s)\left[P M_{s}(s)\right]=} \\
& =V(s)\left[\begin{array}{cccc}
I-D_{u} & C & D_{d} \\
0-B_{u} & A-s I & B_{d}
\end{array}\right]= \\
& =V(s)\left[\begin{array}{ccccc}
I & -D_{u} & C_{x} & C_{z} & D_{d} \\
0-B_{u, x} & A_{x}-s I & A_{12} & B_{d, x} \\
0 & 0 & 0 & A_{z}-s I & 0
\end{array}\right]
\end{aligned}
$$

In the last equality, we have used the assumption of a realization on the form (15). The controllability of the state $x$ from $u$ and $d$ implies, via the PBH test (see (Kailath, 1980)), that the middle block of rows in the matrix $\left[P M_{s}(s)\right]$, has full row-rank for all $s$. Also, $A_{z}$ has full row-rank because of the assumption that the state $z$ is asymptotically stable. Therefore, the matrix $\left[P M_{s}(s)\right]$ has full row-rank for $s=0$. Since $V(s)$ is irreducible, it has also full row-rank for $s=0$. This implies that $W(0)$ has full row-rank.

Now consider the relation

$$
\begin{gathered}
W(s)\left[\begin{array}{c}
L(s) \\
0
\end{array}\right]=V(s) P\left[\begin{array}{c}
L(s) \\
0
\end{array}\right]=V(s)\left[\begin{array}{c}
L(s) \\
0
\end{array}\right]= \\
=V(s)\left[\begin{array}{c}
C(s I-A)^{-1} B_{f}+D_{f} \\
0
\end{array}\right]= \\
=\left[\begin{array}{ll}
V_{1}(s) & V_{1}(s) C(s I-A)^{-1}
\end{array}\right]\left[\begin{array}{l}
D_{f} \\
B_{f}
\end{array}\right]=V(s)\left[\begin{array}{l}
D_{f} \\
B_{f}
\end{array}\right]
\end{gathered}
$$

The last equality follows from the fact that $V(s) M_{s}(s)=0$. The above relation implies that

$$
\left.\left(W(s)\left[\begin{array}{c}
L(s) \\
0
\end{array}\right]\right)\right|_{s \rightarrow 0}=\left.0 \Longleftrightarrow\left(V(s)\left[\begin{array}{l}
D_{f} \\
B_{f}
\end{array}\right]\right)\right|_{s \rightarrow 0}=0
$$

This equality together with the fact that $W(0)$ has full row-rank, implies that we can apply Lemma 1, which proves the theorem.

\subsection{Using the Chow-Willsky Scheme}

The criterion for strong fault detectability, corresponding to Theorem 4, becomes as follows:

Theorem 7. A fault is strongly detectable if and only if

$$
N_{R H} \mathcal{P}_{n} \mu \neq 0
$$

where $N_{R H}$ is a basis for the left null space of $\left[\mathcal{R}_{n} \mathcal{H}_{n}\right]$ and $\mu=\left[\begin{array}{lll}1 & 0 & \ldots\end{array}\right]^{T}$.

In the proof of this theorem, we will use the notation $\Psi_{i}(s)=\left[\begin{array}{lllll}I_{i} & s I_{i} & \ldots & s^{\rho} I_{i}\end{array}\right]^{T}$. Further, we need the notion of primary dependent rows (Kailath, 1980), and the following lemma.
Lemma 2. Assume the rows of the matrix $W$ define the largest and uppermost set of primary dependent rows in $\left[\mathcal{R}_{n} \mathcal{H}_{n}\right]$. Then $F(s)=W\left[\Psi_{m}(s)-\mathcal{Q}_{n} \Psi_{k_{u}}(s)\right]$ is a polynomial basis for $\mathcal{N}\{M(s)\}$ and $F(0)=$ $W\left[\Psi_{m}(0)-\mathcal{Q}_{n} \Psi_{k_{u}}(0)\right]$ has full row rank.

The proof can be found in (Nyberg, 1999). Now return to the proof of Theorem 7:

PROOF. We will start with the only-if part of the proof and an indirect proof is used. Therefore assume that

$$
N_{R H} \mathcal{P}_{n} \mu=0
$$

Let the rows of a matrix $W$ define the largest and uppermost set of primary dependent rows in $\left[\begin{array}{ll}\mathcal{R}_{n} & \mathcal{H}_{n}\end{array}\right]$. Then according to Lemma $2, F(s)=$ $W\left[\Psi_{m}(s)-\mathcal{Q}_{n} \Psi_{k_{u}}(s)\right]$ becomes a polynomial basis for $\mathcal{N}_{L}\{M(s)\}$ and $F(0)$ has full row rank.

Define $X(s)$ as $X(s)=\sum_{i=1}^{\infty} s^{-i} A^{i-1} B_{f}$. Then it can be shown that $\Psi_{m}(s) L(s) \stackrel{=1}{=} \mathcal{R}_{n} X(s)+\mathcal{P}_{n} \Psi_{1}(s)$. Now assume that (17) holds. This implies the following:

$$
\begin{gathered}
\left.\left(F(s)\left[\begin{array}{c}
L(s) \\
0
\end{array}\right]\right)\right|_{s \rightarrow 0}= \\
=\left(W\left[\Psi_{m}(s)-\mathcal{Q}_{n} \Psi_{k_{u}}(s)\right]\left[\begin{array}{c}
L(s) \\
0
\end{array}\right]\right)_{s \rightarrow 0}= \\
=\left(W \Psi_{m}(s) L(s)\right)_{s \rightarrow 0}= \\
=\left(W\left(\mathcal{R}_{n} X(s)+\mathcal{P}_{n} \Psi_{1}(s)\right)\right)_{s \rightarrow 0}={ }^{*} \\
={ }^{*} W \mathcal{P}_{n} \Psi_{1}(0)=W \mathcal{P}_{n} \mu=0
\end{gathered}
$$

The equality marked $={ }^{*}$ holds since $W\left[\mathcal{R}_{n} \mathcal{H}_{n}\right]=0$ and the last equality holds because of (17). Since $F(s)$ is a polynomial basis for $\mathcal{N}_{L}\{M(s)\}$ and $F(0)$ has full row rank, Lemma 1 implies that the fault is not strongly detectable. Thus the only-if part of the proof has been shown.

Also for the if part, an indirect proof will be used. Therefore we assume that the fault is not strongly detectable and want to prove that (17) holds. Assume that $w_{1}$ is an arbitrary row-vector in $N_{R H}$ which means that $w_{1}\left[\mathcal{R}_{n} \mathcal{H}_{n}\right]=0$. Pick other $w_{i}:$ s so that $W=\left[w_{1}^{T} w_{2}^{T} \ldots\right]^{T}$ defines a set of primary dependent rows in $\left[\mathcal{R}_{n} \mathcal{H}_{n}\right]$. This implies that $W\left[\mathcal{R}_{n} \mathcal{H}_{n}\right]=0$ and according to Lemma 2, $F(s)=W\left[\Psi_{m}(s)-\mathcal{Q}_{n} \Psi_{k_{u}}(s)\right]$ becomes a polynomial basis for $\mathcal{N}_{L}\{M(s)\}$. Then we know that for some polynomial matrix $\phi(s)$, it holds that $F(s)=$ $\phi(s) N_{M}(s)$, where $N_{M}(s)$ is a minimal polynomial basis for $\mathcal{N}_{L}\{M(s)\}$. Theorem 5 together with the assumption that the fault is not strongly detectable implies that

$$
\begin{array}{r}
\left(F(s)\left[\begin{array}{c}
L(s) \\
0
\end{array}\right]\right)_{s \rightarrow 0}=\left(\phi(s) N_{M}(s)\left[\begin{array}{c}
L(s) \\
0
\end{array}\right]\right)_{s \rightarrow 0}= \\
=\phi(0)\left(N_{M}(s)\left[\begin{array}{c}
L(s) \\
0
\end{array}\right]\right)_{s \rightarrow 0}=0
\end{array}
$$

Also we have that 


$$
\begin{gathered}
\left(F(s)\left[\begin{array}{c}
L(s) \\
0
\end{array}\right]\right)_{s \rightarrow 0}= \\
=\left(W\left[\Psi_{m}(s)-\mathcal{Q}_{n} \Psi_{k_{u}}(s)\right]\left[\begin{array}{c}
L(s) \\
0
\end{array}\right]\right)_{s \rightarrow 0}= \\
=\left(W \Psi_{m}(s) L(s)\right)_{s \rightarrow 0}= \\
=\left(W\left(\mathcal{R}_{n} X(s)+\mathcal{P}_{n} \Psi_{1}(s)\right)\right)_{s \rightarrow 0}= \\
=W \mathcal{P}_{n} \Psi_{1}(0)=W \mathcal{P}_{n} \mu=0
\end{gathered}
$$

This implies that $w_{1} \mathcal{P}_{n} \mu=0$ which proves the if part, and completes the proof.

Note that only constant matrices are involved in Theorem 7 which implies that the condition (16) can also be written $\operatorname{Im} \mathcal{P}_{n} \mu \nsubseteq \operatorname{Im}\left[\mathcal{R}_{n} \mathcal{H}_{n}\right]$.

\section{DISCUSSION AND CONCLUSIONS}

In this paper, criterions for fault detectability and strong fault detectability, seen as system properties, have been derived. Two new criterions for fault detectability and three new criterions for strong fault detectability, have been presented. In addition, existing criterions have been reviewed. To the authors knowledge, comparable criterions for strong fault detectability have not been presented elsewhere.

Criterions for models given both on transfer function form and state-space form have been considered. All the proofs, for the different criterions, become quite simple thanks to a notion of polynomial bases for linear residual generators. For the case of strong fault detectability, it is shown that the existence of poles in the origin, can not be used, neither as a necessary nor sufficient condition.

These new criterions, together with the already known, give the user several choices to be used in different situations. Below, the criterions are compared, and advice is given on what criterion to use in different situations.

\subsection{Detectability}

If the system model is given on transfer function form and we want to check fault detectability, then the easiest approach is the "frequency domain approach", i.e. the criterion given by Theorem 2 . The reason is that, compared to the "intuitive approach", we do not need to care about the transfer function $G(s)$. The actual test can be performed by either calculating the null space or to perform a rank test. However, the rank test should be the preferred choice since, calculating the rank is numerically a simpler operation than to calculate the null space.

If the system model is given on state-space form and we want to check fault detectability, the best choice is the criterion based on the system matrix, i.e. Theorem 3. The reason for this is that, compared with the Chow-Willsky scheme, the criterion based on the system matrix has shown to be much more numerical stable. However note that the criterion based on the Chow-Willsky scheme, i.e. Theorem 4, uses only constant matrices, in contrast to the criterion based on the system matrix. This might in some cases be an advantage since we do not need special algorithms that can handle polynomial matrices. No matter what the preferred criterion is, in both cases, the actual test is again most easily performed via the rank test.

\subsection{Strong Detectability}

If the system model is given on transfer function form and we want to check strong fault detectability, there is only one alternative. Since the "frequency domain approach" doesn't work we have to use the "intuitive approach", i.e. Theorem 5 . As shown in the previous section, we also have to perform the test by calculating the null space.

Finally, if the system model is given on state-space form and we want to check strong fault detectability, the criterion based on the system matrix, i.e. Theorem 6 , is the best choice. The reason is again the numerical considerations. However an advantage with the criterion based on the Chow-Willsky scheme, i.e. Theorem 7 , is that only constant matrices are needed and also that the rank test is possible to perform. That is, we do not need to calculate a null-space.

\section{REFERENCES}

Chen, Chi-Tsong (1984). Linear System Theory and Design. Holt, Rinehart and Winston, New York.

Chen, J. and R.J. Patton (1994). A re-examination of fault detectability and isolability in linear dynamic systems. Fault Detection, Supervision and Safety for Technical Processes. IFAC. Espoo, Finland. pp. 567-573.

Chow, E.Y. and A.S. Willsky (1984). Analytical redundancy and the design of robust failure detection systems. IEEE Trans. on Automatic Control 29(7), 603-614.

Ding, X. and P.M. Frank (1991). Frequency domain approach and threshold selector for robust model-based fault detection and isolation. IFAC Fault Detection, Supervision and Safety for Technical Processes. Baden-Baden, Germany. pp. $271-276$.

Forney, G.D. (1975). Minimal bases of rational vector spaces, with applications to multivariable linear systems. SIAM J. Control 13(3), 493-520.

Frank, P.M. (1990). Fault diagnosis in dynamic systems using analytical and knowledge-based redundancy - a survey and some new results. Automatica 26(3), 459-474.

Kailath, Thomas (1980). Linear Systems. PrenticeHall.

Magni, J.F. and P. Mouyon (1994). On residual generation by observer and parity space approaches. IEEE Trans. on Automatic Control 39(2), 441447.

Nyberg, M. and E. Frisk (1999). A minimal polynomial basis solution to residual generation for fault diagnosis in linear systems. Vol. P of IFAC. Beijing, China. pp. 61-66.

Nyberg, M. and L. Nielsen (1997). Parity functions as universal residual generators and tool for fault detectability analysis. IEEE Conf. on Decision and Control. pp. 4483-4489.

Nyberg, Mattias (1999). Model Based Fault Diagnosis: Methods, Theory, and Automotive Engine Applications. PhD thesis. Linköping University. URL: http://www.fs.isy.liu.se/Publications/.

Rosenbrock, H.H. (1970). State-Space and Multivariable Theory. Wiley, New York.

The

Polynomial

Toolbox 2.0 for Matlab 5 (1998). Polyx, Czech Republic. URL: http://www.polyx.com. 